\title{
Expanding Mars Science Return in the MSR Era: The Need for, Capabilities of, and Challenges Associated with Small Mars Science Missions
}

\section{Primary Author:}

James M. Kaufman

W: 626-873-7704 C: 626-660-6577

The Aerospace Corporation

james.m.kaufman@aero.org

\section{Co-Authors:}

Don Banfield, Cornell University

Justin S. Boland, NASA/JPL

Matthew A. Eby, The Aerospace Corporation

Paul Hayne, University of Colorado

Jeffrey A. Lang, The Aerospace Corporation

Michael A. Mischna, NASA/JPL

Nilton Renno, University of Michigan

Robert L Staehle, NASA/JPL

Carol R. Stoker, NASA/ARC

Sam W. Thurman, NASA/JPL

Manuel de la Torre Juarez, NASA/JPL

Chris R. Webster, NASA/JPL

Rebecca M. E. Williams, Planetary Science Institute

\section{Co-Signers:}

Sheridan E Ackiss, The Aerospace Corporation

Andrew C. Cortopassi, The Aerospace Corporation

Jerry K. Fuller, The Aerospace Corporation

Robert Grimm, SwRI 


\title{
Expanding Mars Science Return in the MSR Era: The Need for, Capabilities of, and Challenges Associated with Small Mars Science Missions
}

\author{
POC: James M. Kaufman ${ }^{1}$ (james.m.kaufman@aero.org, W: 626-873-7704 C: 626-660-6577) \\ Don Banfield ${ }^{2}$, Justin S. Boland ${ }^{3}$, Matthew A. Eby ${ }^{1}$, Paul Hayne ${ }^{4}$, Jeffrey A. Lang ${ }^{1}$, Michael A. Mischna ${ }^{3}$ \\ Nilton Renno ${ }^{5}$, Robert L Staehle ${ }^{3}$, Carol R. Stoker ${ }^{6}$, Sam W. Thurman ${ }^{3}$, Manuel de la Torre Juarez ${ }^{3}$, \\ Chris R. Webster ${ }^{3}$, Rebecca M. E. Williams ${ }^{7}$ \\ ${ }^{1}$ The Aerospace Corporation, ${ }^{2}$ Cornell, ${ }^{3}$ NASA/JPL, ${ }^{4} \mathrm{U}$. Colorado, ${ }^{5} \mathrm{U}$. Mich., ${ }^{6}$ NASA/ARC, ${ }^{7} \mathrm{PSI}$
}

\begin{abstract}
This paper advocates for NASA to create and fund a class of small Mars missions (\$125M - \$250M), along with a non-instrument technology maturation program, to complement the Mars Sample Return (MSR) program. Arguments are provided that without such a program, Mars science missions not related to Mars sample return will be put on hold for a decade or more.

\section{Introduction}

Given the likelihood of Mars Sample Return (MSR) being the dominant Mars exploration effort over the next decade and beyond, the Mars science community could find itself in a situation similar to what the astrophysics community has been in for over a decade. Namely, that a large, multi-\$B mission (James Webb Space Telescope, JWST) consumed most available resources at the expense of other valuable science investigations, along with the associated loss of expertise. Hence, there is a need for smaller programs to maintain a diverse Mars exploration science portfolio during the MSR Era (following the astrophysics analogy) with small, i.e., SMEX- or MIDEX-class (\$125M \$250M) Mars missions.
\end{abstract}

Note that we are assuming that NASA will still maintain a vigorous Mars Exploration Program (MEP) and that any rebalancing of funding will remain within MEP. We believe that maintaining a separate MEP is justifiable because of the following:
- Many of the achievable Mars science investigations are directly tied to the question of habitability and life in the solar system

- Preparation for subsequent human exploration of Mars

- Proximity of Mars compared to other bodies means discoveries can be acted upon more rapidly

- Operating in the Mars environment requires less expensive technology developments than would be required for outer planets, ocean worlds, Venus, etc.

\section{Summary of Various Small Mars Mission Architectures \\ Below, we list examples of small Mars mis- sion architectures that have been studied over the last 10 years. For each mission we also list at least one associated technical challenge that must be overcome to make the mission a reality.}

MarsDrop. (1) This unique concept (Figure 1) uses a Reentry Breakup Recorder (2) (REBR)/Deep Space 2 (3) (DS2) heritage entry system combined with a 1960s-era, proven, steerable Rogallo parawing enabling landed access to scientifically interesting, but potentially hazardous Martian terrain. When combined with Terrain Relative Navigation (TRN), this system could potentially achieve pinpoint landing accuracy, i.e. within a few 10 s of meters of the target. 


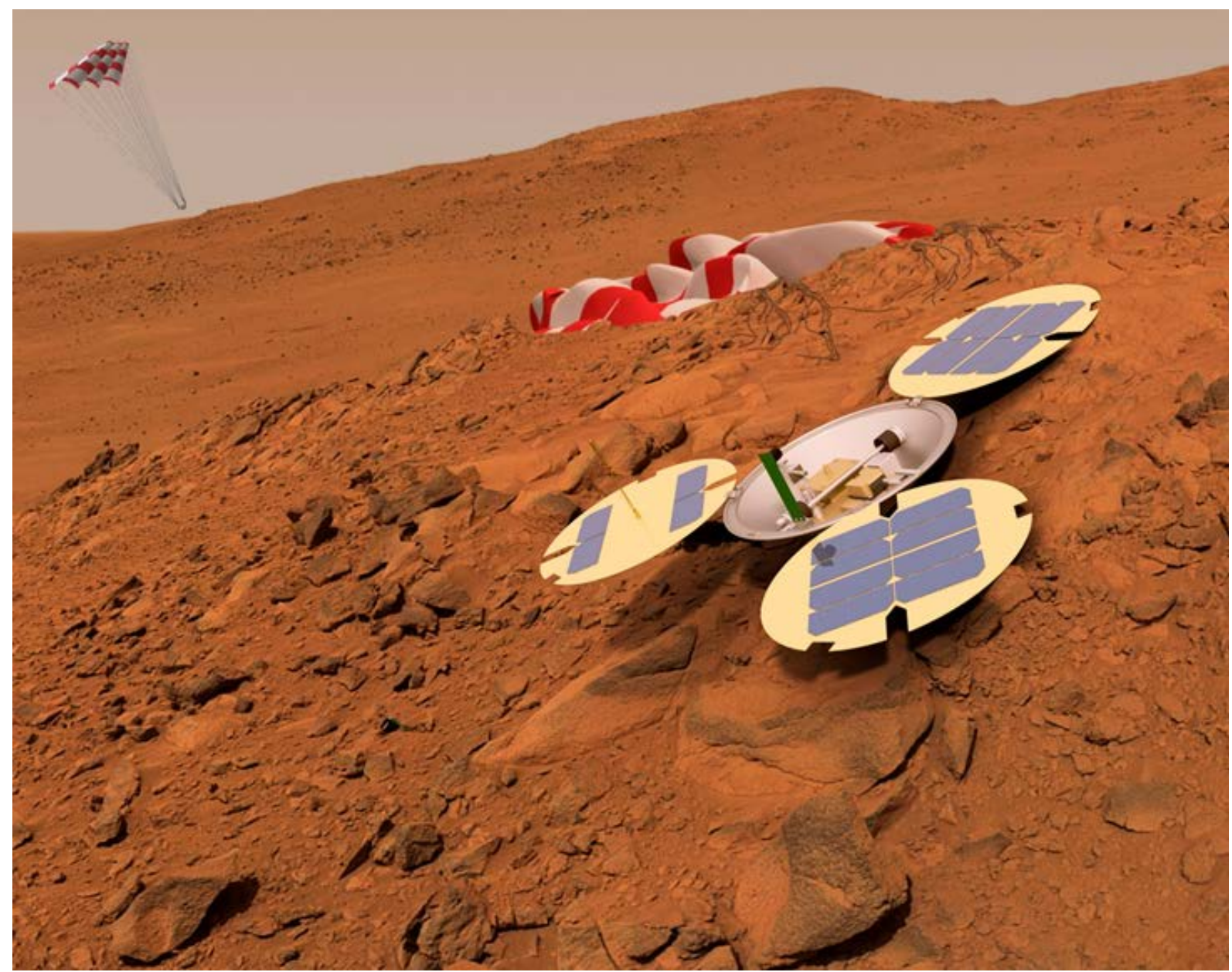

Figure 1. The MarsDrop Concept on Mars. Shown are solar arrays that deploy from the REBR-like entry system and the deflated parawing. The solar arrays are used to right the lander in a manner similar to Mars Pathfinder and the Mars Exploration Rovers. The entire size of the microlander is $\sim 0.6 \mathrm{~m}$ across. MarsDrop was jointly developed by The Aerospace Corporation and NASA/JPL.

Challenge(s): 1) This system combines a number of high-heritage concepts that collectively have no heritage. Therefore, significant system-level terrestrial testing, e.g., high-altitude balloon testing will be required. 2) Stable entry of an entry system using the REBR/DS2 form factor will require a CG that is substantially forward in the forebody, thus complicating the packaging of the internal subsystems and instruments.

Rough Landers. $(4,5)$ This concept results in relative design simplicity (for example, by using balsa wood or some other crushable material) at the cost of higher landing impact load factors (500-1,500 g as compared to 30$50 \mathrm{~g}$ for previously flown Mars landers, but not in the 60,000-g regime DS2 expected to see). Figure 2 shows JPL's Small High Impact Energy Landing Device (SHIELD) rough lander concept.

Challenge(s): 1) The relatively high landing impact load will transfer design issues to the subsystems and instruments. 2) Lack of heritage makes this concept riskier than MarsDrop.

Low-Ballistic-Coefficient Entry Systems. These concepts replace the traditional heat shield/parachute concept with an inflatable and possibly deformable drag device (to allow for aerodynamic steering). There have been several high-profile, mainly successful 


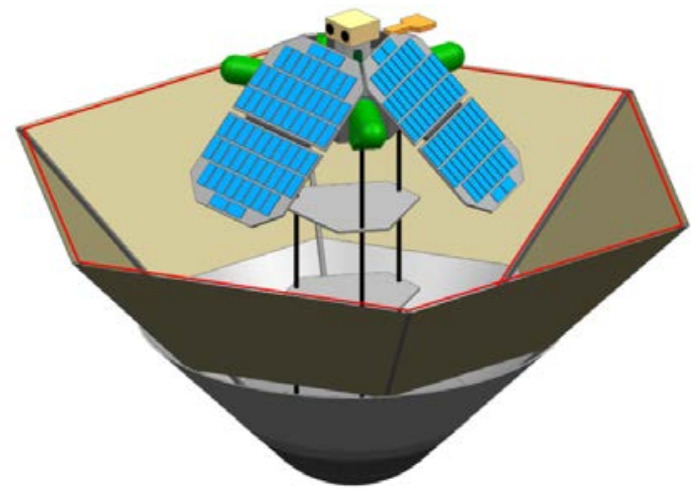

Figure 2. The SHIELD Rough Lander Concept. Developed by JPL, Small High Impact Energy Landing Device (SHIELD) uses a crushable landing system combined with a drag skirt to achieve a landing impact load factor of $~ 1000 \mathrm{~g}$.

demonstrations of these concepts: Hypersonic Inflatable Aerodynamic Decelerator (6, 7) (HIAD), and Low-Density Supersonic Decelerator (8) (LDSD) (Figure 3).

Challenges(s): Although HIAD and LDSD have been successfully flight tested, thus resulting in relatively high TRLs covering the deceleration from interplanetary speeds to survivable landing speeds, they have not yet demonstrated the ability to land on solid ground, i.e., they have not yet demonstrated the ability to provide a landed platform from which to successfully make viable science measurements. With additional development and testing, we believe that these concepts will one day enable landed science missions
CubeSat Missions. There is no argument that CubeSat missions have skyrocketed in popularity and have generated high-quality science results and/or served as technology demonstration vehicle. Indeed, the first interplanetary CubeSat mission was Mars Cube One (9) (MarCO), a twin CubeSat mission developed to relay critical events data from the InSight (Interior Exploration using Seismic Investigations, Geodesy and Heat Transport) lander mission's EDL phase to Earth. Other planetary science CubeSat missions are currently in development, such as Lunar Flashlight (10) (LF) and Near-Earth Asteroid Scout (11) (NEAScout).

Challenge(s): CubeSats were originally designed to serve as a low-cost opportunity to provide college students with hands-on experience in developing a spaceflight mission through its entire lifecycle. One of the hallmarks of the CubeSat concept that keeps the cost low also limits the type of science payloads that can be accommodated, namely, that they must be designed to the well-defined, but constraining CubeSat format of multiples of $10 \mathrm{~cm} \times 10 \mathrm{~cm} \times 10 \mathrm{~cm}$ cubes to maximize the number of rideshare launch opportunities by using a common deployment mechanism.

SmallSat Missions (Non-CubeSats). Here, we refer to small spacecraft equivalent to

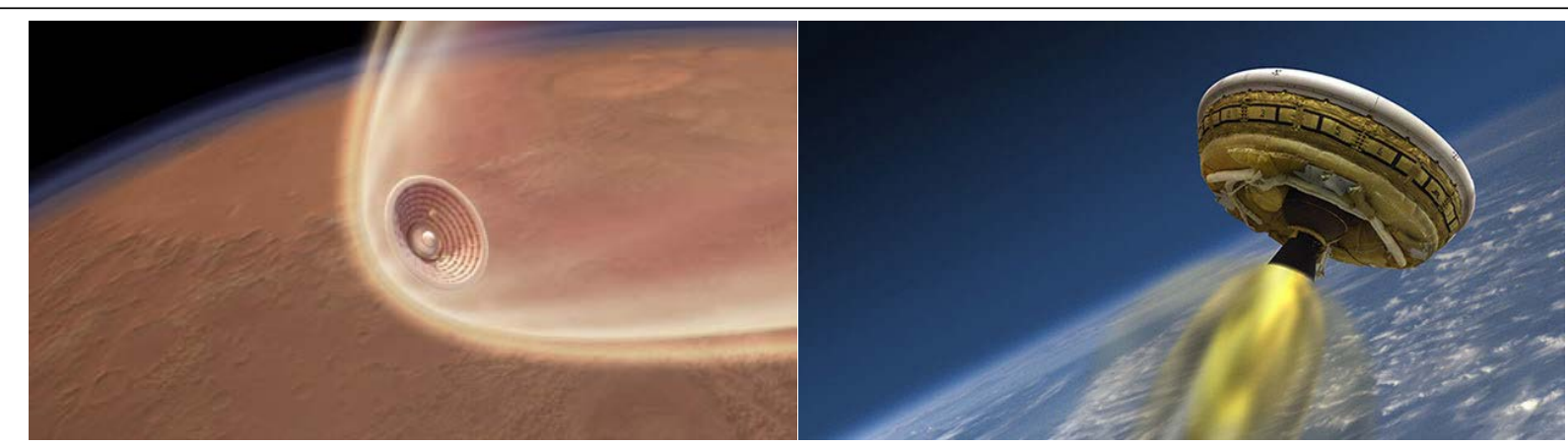

Figure 3. Examples of Low-Ballistic-Coefficient Entry Systems. NASA/LaRC's Hypersonic Inflatable Aerodynamic Decelerator (HIAD) (L) and NASA/JPL's Low-Density Supersonic Decelerator (LDSD) (R). Both of these systems have been extensively tested using sounding rockets, high-altitude balloons, rocket sleds, and wind tunnels. While each has proven sound as an atmospheric decelerator, all flight testing has ended with water landings. No viable system for landing on solid ground has yet been developed. 
approximately 6U-24U CubeSats, but not relying on the CubeSat form factor. Here, we are referring to "traditional" spacecraft architectures that are similar to current missions such as Mars Odyssey, MRO, MAVEN, etc., only roughly an order of magnitude smaller in size, mass, and cost. This is accomplished partially through the use of organic reductions in mass, power, volume, etc., requirements of typical spacecraft subsystems, and through more targeted reductions in resource requirements. Several such mission concepts are described by Barba, et al. (2020) (5).

Challenge(s): The primary impediment to implementing missions of this class is more programmatic than technical in nature. In many situations, technologies exist that would enable missions of this class, however, due to NASA's general aversion to risk and the desire to always fly hardware with extensive heritage, these missions can never really materialize. If the technologies could either be matured or demonstrated (as required), then missions of this class would be more programmatically feasible.

\section{Examples of Mars Science Investigations Enabled by Small Missions}

A wide range of science objectives could benefit from these small Mars mission architectures including the following, with references to investigations listed in the new MEPAG Goals document (12):

- Determining if time-varying methane signatures are biogenic or non-biogenic in nature. [I B1.2, II A3.1]

- Understanding saltation and the effects of electrostatics (also relevant to future human exploration of Mars). [II A2.1, III A2.5, IV B2.1, IV B3.1, IV B4.3]

- The nature of Mars' local diurnal hydrological cycle and deliquescence. [II A2, III A1.1]
- Understanding Mars’ climate variation using records contained within polar layered deposits (PLD). [II A2, II B1, III A1.3]

- The search for water sources, e.g., in situ measurements at recurring slope lineae (RSL), candidate human exploration sites, etc. [I A2.1, III A1.1, III A4.3, IV A3, IV C2, IV D1.1]

- Determining Mars’ geologic history from vertical exposures, i.e., crater walls, pits, chasms (e.g., Valles Marineris), etc. [III A3.1, III A4.5, III A4.7]

- Direct measurements of Mars winds/temperature/humidity/surface pressure using a network of mutually occulting satellites. [II A, IV B3, IV C2]

- Continuous monitoring of weather and documenting the temporal behavior of dust storm genesis on time scales shorter than one sol from areo-stationary orbit. [II A, III A2.4]

- In situ landing site characterization for human exploration. [IV A3, IV B, IV D2]

- Characterization of the Martian interior via deployment of a seismometer network. [III B1.2, III B2.1]

- Monitoring of modern surface processes with increased temporal monitoring from surface-based observations. [III A4.3]

All of these objectives could be met through the use of one or more of the above architectures, combined with new or maturing instruments. Currently, however, there are limited Mars launch opportunities, especially for comanifested or ride-along payloads. NASA's Small Innovative Missions for Planetary Exploration (SIMPLEx) program is designed to provide such launch opportunities, however, SIMPLEx relies on commercial GEO launches and the limited number of NASA 
deep-space launches, neither of which will necessarily get a small mission to Mars.

Increasing the allowable propulsive capability of SIMPLEx missions, the number of appropriate and allowable ride-along missions and/or co-manifested launch opportunities, and the use of small launch vehicles that could place a small payload on a direct-toMars trajectory (combined with a small, high-efficiency transfer stage) would greatly open up the opportunity space for small Mars science missions. Of course, a commensurate increase in funding would also be required.

Compounding the lack of launch opportunities is the need for technology maturation associated with:

- Small propulsion systems capable of $\sim 1-2$ $\mathrm{km} / \mathrm{sec}$ of $\Delta \mathrm{V}(\sim 10 \mathrm{X}$ greater than current capabilities)

- Novel propulsion systems that make use of thermal runaway conditions in Lithium ion batteries to provide thrust. Such systems could be used after the batteries are no longer required, e.g., doing a retropropulsive burn from Mars to deploy a small lander.

- Flight-proven propulsive EELV Secondary Payload Adapter (ESPA) rings

- Small entry systems (including those with and without REBR/DS2 heritage)

- Inflatable/deployable entry systems and other drag devices

- Steerable parawings combined with Terrain Relative Navigation (TRN) to achieve pin-point landings

- Non propulsive guidance systems for use in EDL, e.g., CG-shifting, deformable aerodynamic systems, control surfaces, etc. Such systems may not increase EDL accuracy, but may allow for a higher payload-to-total-mass ratio.
- Miniaturized C\&DH, telecom, and other spacecraft subsystems

- Use of increased amounts of autonomy and/or AI combined with new higher performance C\&DH systems to increase science output with limited telecom resources

We therefore advocate the need for a balanced Mars science exploration program during the MSR Era: NASA should create a new, small Mars mission program within MEP chartered with developing SMEX-toMIDEX-sized Mars missions (either launched directly by NASA, or as ridealongs or co-manifested payloads on military, commercial, and foreign missions/launch vehicles).

This should be further supported with a Mars-specific, non-instrument, technology maturation program for small orbiting and landed spacecraft architectures funded through a new ROSES Program Element Appendix.

Note that simply arguing one could propose a low-cost Mars mission to the Discovery Program is not a viable option for the following reasons:

- The small Mars missions would be in competition against other solar system targets, each supported by equally important community science roadmaps

- Discovery is historically extremely risk averse and the use of new technologies to minimize cost would not pass muster with a TMC evaluation team (hence further justifying the need for a non-instrument technology maturation program which could benefit both Mars and other planetary missions)

Thus, the need for need for a small Mars mission program augmented with sufficient technology development funding still remains. 


\section{References}

1. Multiplying Mars Lander Opportunities with MARSDROP Microlanders. R. L. Staehle, S. Spangelo, M. S. Lane, K. M. Aaron, R. Bhartia, J. S. Boland, L. E. Christensen, S. Forouhar, M. de la Torre Juarez, N. Trawny, C. R. Webster, M. A. Eby, R. M. E. Williams, D. A. Paige. 29th Annual AIAA/USU Conference on Small Satellites, August 8-13, 2015, Logan, UT, USA.

2. Spacecraft Reentry Breakup Recorder, W. H. Ailor, R. J. Bywater, L. Gurevich. US Patent 6,895,314. The Aerospace Corporation, El Segundo, CA. May 17, 2005.

3. Deep Space 2: The Mars Microprobe Mission. S. Smrekar, et al., J. Geophys. Res., 104(E11), 27,013-27,030, 1999.

4. Rough Lander Concept for Mar Exploration. S. W. Thurman and T. P. Rivellini, 2002 Core Technologies for Space Systems Conference, Colorado Springs, CO, USA.

5. Exploring Mars With Small Spacecraft Orbiters and Landers. N. J. Barba, T.A. Komarek, V. Stamenković, L. Giersch, R. C. Woolley, C. D. Edwards. 51st Lunar and Planetary Science Conference, March 16-20, 2020, The Woodlands, TX, USA.

6. The HIAD Orbital Flight Demonstration Instrumentation Suite. G. Swanson, B. Smith, R. Akamine, R. J. Bodkin, N. Cheatwood, S. Hughes, D. Gaddy, A. Parker. 15th International Planetary Probe Workshop, June 11-15, 2018, Boulder, CO, USA.

7. Hypersonic Inflatable Aerodynamic Decelerator (HIAD) Technology Development Overview. S. J. Hughes, F. M. Cheatwood, R. A. Dillman, H S. Wright, J. A. DelCorso, A. M. Calomino. 21st
AIAA Aerodynamic Decelerator Systems Technology Conference and Seminar, 2326 May 2011, Dublin, Ireland.

8. Summary of the First High-Altitude, Supersonic Flight Dynamics Test for the Low-Density Supersonic Decelerator Project. I. G. Clark, M. Adler, R. Manning. 23rd AIAA Aerodynamic Decelerator Systems Technology Conference, Mar. 30-Apr. 2, 2015, Daytona Beach, FL, USA.

9. MarCO: Interplanetary Mission Development on a CubeSat Scale. J.

Schoolcraft, A. T. Klesh, T. Werne. AIAA 2016-2491,Session: SSCSO SmallSat Missions \& Operations I, SpaceOps 2016 Conference, May 16-20, 2016, Daejeon, Korea.

10. Lunar Flashlight: Illuminating the Moon's South Pole. P. O. Hayne, B. A. Cohen, B. T. Greenhagen, D. A. Paige, J. M. Camacho, R. G. Sellar, J. Reiter. 47th Lunar and Planetary Science Conference, March 21-25, 2016, The Woodlands, TX, USA.

11. Near Earth Asteroid (NEA) Scout. L. Johnson, J. Castillo-Rogez, J. Dervan, L. McNutt. 4th International Symposium on Solar Sailing (ISSS 2017), January 17-20, 2017, Kyoto, Japan.

12. Mars Scientific Goals, Objectives, Investigations, and Priorities: 2020. D. Banfield, ed., 89 p. White Paper posted March, 2020 by the Mars Exploration Program Analysis Group (MEPAG) at https://mepag.jpl.nasa.gov/reports.cfm. 